\title{
Treatment of lupus erythematosus of the eyelids with pulsed dye laser
}

\author{
Susana Brás ${ }^{1}$ (D) Borja Gonzalez ${ }^{2}$ - Gonzalo Segurado-Miravalles ${ }^{3} \cdot$ Pablo Boixeda $^{4}$
}

Received: 23 May 2016 / Accepted: 14 December 2016

(C) Springer-Verlag London 2016

\section{Background}

Cutaneous lupus erythematosus (CLE) is an autoimmune disease with heterogeneous clinical features that can be divided in acute (ACLE), subacute (SCLE), and chronic (CCLE) [1]. This classification is based on a combination of clinical features, histological changes, laboratory abnormalities, and average duration of skin lesions [2].

CCLE is the most prevalent form of CLE [1], including several subtypes. Discoid lupus erythematosus (DLE) is the most common form of CCLE, generally involving the face (forehead, philtrum, malar region, nose, ears, and cheek) in a longstanding and disfiguring way [3].

DLE involving the eyelids is rare and it may precede the development of DLE in other skin locations [4, 5]. Blepharitis and infiltrated erythematous plaques, with skin atrophy, scarring, and telangiectasia are most frequently observed [3, 4]. The lower eyelid is also commonly affected, usually with loss of eyelashes (madarosis) [3].

The research was conducted in the Department of Dermatology of the Hospital Universitario Ramon y Cajal, Madrid, Spain.

Susana Brás

bras.susana@gmail.com

1 Dermatology Department, Hospital de Santo António dos Capuchos, Centro Hospitalar de Lisboa Central, Lisbon, Portugal

2 Dermatology Department, Hospital Universitario Insular de Gran Canaria, Las Palmas, Spain

3 Dermatology Department, Hospital Universitario Ramon y Cajal, Madrid, Spain

4 Laser Unit, Dermatology Department, Hospital Universitario Ramon y Cajal, Madrid, Spain
Standard medical therapies include patient education on proper sun protection along with topical corticosteroids or oral antimalarial drugs. Topical tacrolimus and pimecrolimus have also described as effective [3, 4]. If lack of response occurs, other systemic immunosuppressive drugs may be tried [6].

Lasers have been described as successful in treating different types of CLE, mostly DLE. Henderson et al. reported a case using CO2 laser in 1986 [7] and Zachariae firstly used argon laser to treat DLE telangiectasias [8]. The use of pulsed dye laser (PDL) to treat DLE was described in 1995 by Núñez et al. [9]; since then, it has been used in several forms of CLE with clearance rates higher than $60 \%$ [9-14].

PDL improves the telangiectasic component, erythema, and scaling. Histologic examination comparing before and after PDL treatment shows reduction in the dermal lymphocytic infiltrate, more evident in the superficial papillary dermis and improvement in basal cell hydropic degeneration and pigment incontinence. A reduction of the expression of cutaneous inflammatory regulators such as intercellular adhesion molecule-1 (ICAM-1) and vascular cell adhesion molecule-1 (VCAM-1) was also observed [10]. Cosmetically satisfactory results with few side effects (transient hipo/hyperpigmentation and slight scarring) are reported in most cases $[9,11-13,15,16]$ and recurrence after laser treatment is rare ( 3 of 14 patients with a median of 10 months follow-up) [13].

The authors report a series of three cases, wishing to discuss the efficacy and safety of 595-nm PDL for the treatment of DLE of the eyelids.

\section{Material and methods}

Three female patients with at least one lesion of DLE located on the eyelids were treated with PDL (Table 1). All patients gave their informed consent prior to their inclusion in the 


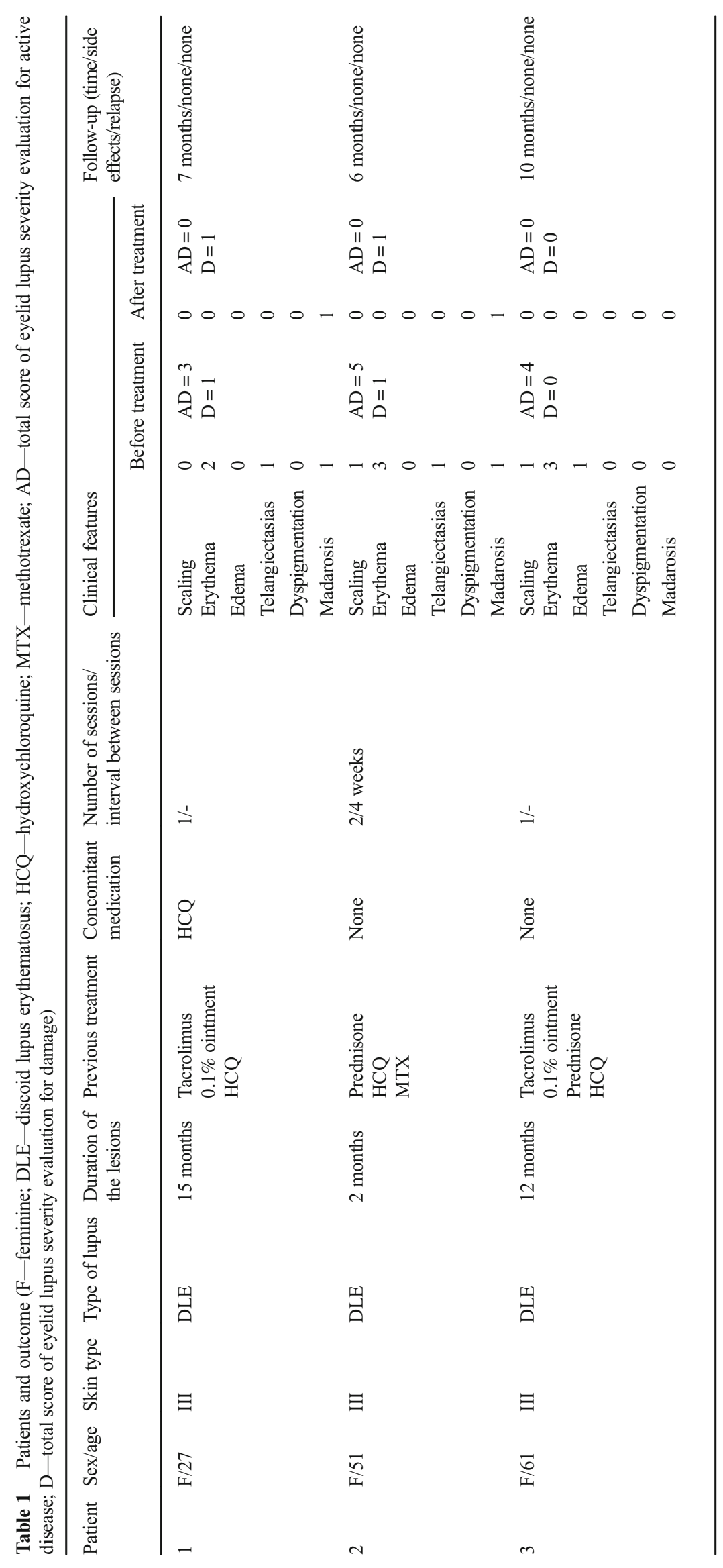


Table 2 Eyelid cutaneous lupus erythematosus severity score

\begin{tabular}{lll}
\hline Evaluation of active disease & $\begin{array}{l}\text { Scaling } \\
\text { Erythema }\end{array}$ & $\begin{array}{c}\text { Absent (0); scale (1); verrucous, hypertrophic (2) } \\
\text { Absent (0); pink, faint erythema (1); red (2); dark red, } \\
\text { purple, violaceous, crusted, hemorrhagic (3) }\end{array}$ \\
& Edema & Absent (0); present (1) \\
& Telangiectasias & Absent (0); present (1) \\
Evaluation of damage & Dyspigmentation & Absent (0); present (1) \\
& Madarosis & Absent (0); present (1) \\
\hline
\end{tabular}

study. Clinical diagnosis was supported by histology and direct immunofluorescence. Two of the patients had lesions only on the eyelids and one had a long history of refractory DLE involving her face and hands. All patients lacked clinical response to previous treatments and were recommended strict sun avoidance, use of sunglasses and topical sunscreens (SPF 50). Patient 1 was maintained on systemic therapy until the day of the laser treatment.
Fig. 1 Images before treatment (left side) and 4 weeks after first treatment (right side). a, b Refers to patient 1. c, $\mathbf{d}$ Refers to patient 2. e, f Refers to patient 3
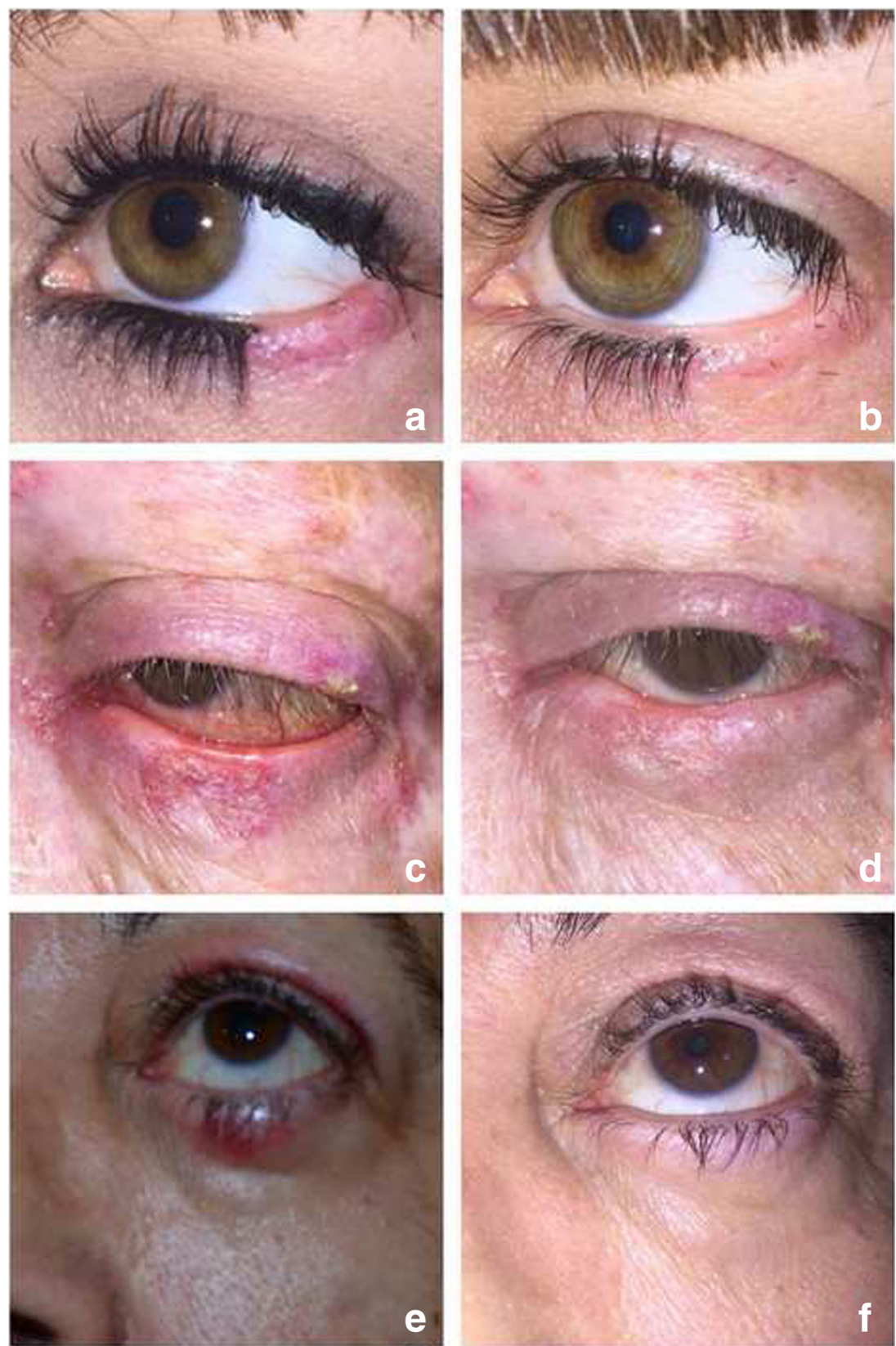
The patients were treated with 595-nm PDL irradiation (Cynergy, Cynosure, Massachusetts, USA) using a $10 \mathrm{~mm}$ spot, a fluency of $8 \mathrm{~J} / \mathrm{cm}^{2}$, and a single pulse of $0.5 \mathrm{~ms}$. Air cooling system was set at level 4 (Cryo 6, Zimmer Medizinsysteme GmbH, Neu-Ulm, Germany) and the lesions were covered with a thin layer of ultrasound gel for epidermal protection. The eyes were protected with an intra-ocular non reflective metal shield (Cox II, Oculo-Plastik, Canada) and the laser was pointed away from the eyeballs. The lesions were treated until a purpuric end point was reached; this occasionally required double passing but pulse stacking was avoided.

Clinical improvement was assessed by comparing digital photographs of each patient taken at baseline and 4 weeks after PDL, using a specific eyelid score (Table 2), adapted from CLE Disease Area and Severity Index (CLASI) [17]. Six different parameters were evaluated: scaling, erythema, edema, telangiectasias (these four corresponding to disease activity), dyspigmentation, and madarosis (these two corresponding to damage).

\section{Results}

The results are shown in Table 1. The treatment was well tolerated with no need for anesthesia or analgesia. An intense purpuric response was immediately observed after PDL treatment. All the lesions responded to the treatment (Fig. 1), with significant improvement of erythema, scaling, edema, and telangiectasias. Madarosis was irreversible in all cases. One patient had a partial response and was retreated using the same parameters with complete resolution of the lesions. No skin or ocular side effects were reported.

\section{Discussion}

All the patients had DLE lesions on the left inferior eyelid, two of them with madarosis. In all cases, PDL was used as an alternative therapy in refractory lesions. One patient had been taking hydroxychloroquine for 7 months with no response until the day of laser therapy; given the rapid resolution of the lesions after PDL therapy, we assume that the laser treatment was the preponderant factor that leads to clinical improvement. One patient had a partial response and was retreated with the same parameters at week 4 , with complete resolution of the lesions at week [8]. After laser treatment, the patients were recommended strict sun avoidance, use of sunglasses, and topical sunscreens (SPF 50). There was no relapse at 6 months follow-up.

Untreated eyelid DLE often leads to scarring, ectropion, and madarosis. We highlight the good clinical response regarding the erythematosus and the telangiectasic component and the lack of effect on the madarosis. Given the rapid improvement after 1 or 2 sessions, we believe that PDL is a valid treatment for active eyelid DLE and it should be offered in an early phase of disease, in order to avoid irreversible damage. Further studies are needed to support this hypothesis.

Ocular complications such as anisocoria, uveitis, pupillary distortion, posterior synechiae, iris atrophy, nuclear cataract, visual field defect, macular hole, and retinal scarring have been reported with Diode laser $(800 \mathrm{~nm})$, Alexandrite laser (755 nm), and Intense Pulsed Light (IPL) (500-1200 nm), mostly related to lack of eye protection [18-21]. Although PDL has a lower tissue penetration (wavelength of $595 \mathrm{~nm}$ ), permanent visual impairment has also been reported [22]. Eye safety procedures should always be followed during periocular laser treatments. The treatment was well tolerated without no anesthesia or analgesia.

Limitations of this study include the limited number of treated patients and the short follow-up period.

\section{Conclusions}

PDL is safe and effective in treating DLE involving the eyelids. Given the significant clinical improvement in this study and the previously published good results of PDL in the treatment of CLE [7, 9-14, 23], we recommend early PDL treatment as an adjunctive therapy in controlling DLE disease activity.

\section{Compliance with ethical standards}

Conflict of interest The authors declare that they have no conflicts of interest.

\section{References}

1. Durosaro O, Davis MDP, Reed KB, Rohlinger AL (2009) Incidence of cutaneous lupus erythematosus, 1965-2005: a population-based study. Arch Dermatol 145(3):249-253

2. Okon LG, Werth VP (2014) Cutaneous lupus erythematosus: diagnosis and treatment. Best Pract Res Clin Rheumatol 27(3):391-404

3. Arrico L, Abbouda A, Abicca I, Malagola R (2015) Ocular complications in cutaneous lupus erythematosus: a systematic review with a meta-analysis of reported cases. J Ophthalmol 2015(254260):1-8

4. Cyran S, Douglass MC, Silverstein JL (1992) Chronic cutaneous lupus erythematosus presenting as periorbital edema and erythema. J Am Acad Dermatol 26(2):334-338

5. Aslam F, Lu L (2013) An unusual eyelid swelling. Q J Med 106(12):1145

6. Yélamos O, Roé E, Baselga E, Puig L (2014) Pediatric cutaneous lupus erythematosus treated with pulsed dye laser. Pediatr Dermatol 31(1):113-115

7. Henderson DL, Odom JC (1986) Laser treatment of discoid lupus (case report). Lasers Surg Med 6:12-15 
8. Zachariae H, Bjerring P, Cramers M (1988) Argon laser treatment of cutaneous vascular lesions in connective tissue diseases. Acta Derm Venereol 68(2):179-182

9. Núnez M, Boixeda P, Miralles ES, de Misa RF, Ledo A (1995) Pulsed dye laser treatment in lupus erythematosus telangiectoides. Br J Dermatol 133:1010-1018

10. Díez MTT, Boixeda P, Moreno C, González JA, Zamorano ML, Olasolo PJ (2011) Histopathology and immunohistochemistry of cutaneous lupus erythematosus after pulsed dye laser treatment. Dermatol Surg 37(7):971-981

11. Núnes M, Boixeda P, Miralles ES, de Misa RF, Ledo A (1996) Pulsed dye laser treatment of telangiectatic chronic erythema of cutaneous lupus erythematosus. Arch Dermatol 132:354-355

12. Gupta G, Roberts T (1999) Pulse dye laser treatment of subacute cutaneous lupus erythematosus. Clin Exp Dermatol 24:498-500

13. Baniandrés O, Boixeda P, Belmar P, Pérez A (2003) Treatment of lupus erythematosus with pulsed dye laser. Lasers Surg Med 32(4): $327-330$

14. Truchuelo MT, Boixeda P, Alcántara J, Moreno C, De Las HE, Olasolo PJ (2012) Pulsed dye laser as an excellent choice of treatment for lupus tumidus: a prospective study. J Eur Acad Dermatol Venereol 26(10):1272-1279

15. Erceg A, Bovenschen HJ, Van de Kerkhof PCM, de Jong EMJG, Seyger MMB (2009) Efficacy and safety of pulsed dye laser treatment for cutaneous discoid lupus erythematosus. J Am Acad Dermatol 60(4):626-632

16. Brauer JA, Gordon Spratt EA, Geronemus RG (2013) Laser therapy in the treatment of connective tissue diseases: a review. Dermatol Surg 1-13

17. Bonilla-Martinez ZL, Albrecht J, Troxel AB et al (2008) The cutaneous lupus erythematosus disease area and severity index. Arch Dermatol 144(2):173-180

18. Parver D, Dreher R (2012) Ocular injury after laser hair reduction treatment to the eyebrow. Arch Opthalmol 130(10):1330-1334

19. Karabela Y, Eliacik M (2015) Anterior uveitis following eyebrow epilation with alexandrite laser. Int Med Case Rep J 8:177-179

20. Hammes S, Augustin A, Raulin C, Ockenfels H-M, Fischer E (2007) Pupil damage after periorbital laser treatment of a portwine stain. Arch Dermatol 143(3):392-394

21. Crabb M, Chan WO, Taranath D, Huilgol SC (2014) Intense pulsed light therapy (IPL) induced iritis following treatment for a medial canthal capillary malformation. Australas J Dermatol 55(4):289-291

22. Smalley PJ (2011) Laser safety: risks, hazards, and control measures. Laser Ther 20(2):95-106

23. Ekbäck MP, Troilius A (2013) Laser therapy for refractory discoid lupus erythematosus when everything else has failed. J Cosmet Laser Ther 15(5):260-265 